\title{
2D SPATIAL FREQUENCY CONSIDERATIONS IN COMPARING 1D POWER SPECTRAL DENSITY MEASUREMENTS ${ }^{*}$
}

\author{
Peter Z. Takacs ${ }^{1}$, Sam Barber ${ }^{2}$, Eugene L. Church ${ }^{1}$, \\ Konstantine Kaznatcheev ${ }^{1}$, Wayne R. McKinney ${ }^{2}$, and Valeriy Y. Yashchuk ${ }^{2}$ \\ ${ }^{1}$ Brookhaven National Laboratory \\ Upton, NY 11973-5000 \\ ${ }^{2}$ Lawrence Berkeley National Laboratory \\ Berkeley, CA 94720
}

February, 2010

\begin{abstract}
*This manuscript has been authored by Brookhaven Science Associates, LLC under Contract No. DC-AC02-98CH10886 with the U.S. Department of Energy. The United States Government retains, and the publisher, by accepting the article for publication, acknowledges, a world-wide license to publish or reproduce the published form of this manuscript, or allow others to do so, for the United States Government purposes.
\end{abstract}




\section{DISCLAIMER}

This work was prepared as an account of work sponsored by an agency of the United States Government. Neither the United States Government nor any agency thereof, nor any of their employees, nor any of their contractors, subcontractors, or their employees, makes any warranty, express or implied, or assumes any legal liability or responsibility for the accuracy, completeness, or any third party's use or the results of such use of any information, apparatus, product, or process disclosed, or represents that its use would not infringe privately owned rights. Reference herein to any specific commercial product, process, or service by trade name, trademark, manufacturer, or otherwise, does not necessarily constitute or imply its endorsement, recommendation, or favoring by the United States Government or any agency thereof or its contractors or subcontractors. The views and opinions of authors expressed herein do not necessarily state or reflect those of the United States Government or any agency thereof. 


\title{
2D Spatial Frequency Considerations in Comparing 1D Power Spectral Density Measurements
}

\author{
Peter Z. Takacs, ${ }^{1 \dagger}$ Sam Barber, ${ }^{2}$ Eugene L. Church, ${ }^{1}$ Konstantine Kaznatcheev, ${ }^{1}$ Wayne R. McKinney, ${ }^{2}$ \\ Valeriy Y. Yashchuk ${ }^{2}$ \\ ${ }^{1}$ Brookhaven National Lab, Upton, NY 11973 \\ ${ }^{2}$ Lawrence Berkeley National Lab, Berkeley, CA 94720 \\ 'Corresponding author: takacs@bnl.gov
}

\begin{abstract}
The frequency footprint of 1D and 2D profiling instruments needs to be carefully considered in comparing 1D surface roughness spectrum measurements made by different instruments. Contributions from orthogonal direction frequency components can not be neglected. OCIS codes: $120.3940,120.6650,120.6660,120.4800,220.4840,340.7470,350.4800$
\end{abstract}

\section{Introduction}

The use of optical profiling instruments is ubiquitous in the measurement of the roughness of optical surfaces. Their ease-of-use and non-contact measurement method found widespread use in the optics industry for measuring the quality of delicate optical surfaces. Computerized digital data acquisition with these instruments allowed for quick and easy calculation of surface roughness statistics, such as root-mean-square (RMS) roughness. The computing power of the desktop computer allowed for the rapid conversion of spatial domain data into the frequency domain, enabling the application of sophisticated signal processing techniques to be applied to the analysis of surface roughness, the most powerful of which is the power spectral density (PSD) function. Application of the PSD function to surface statistics introduced the concept of "bandwidth-limited" roughness, where the value of the RMS roughness depends critically upon the spatial frequency response of the instrument. Different instruments with different spatial frequency response characteristics give different answers when measuring the same surface[1].

The earliest optical profilometers used linear array sensors and were, by nature, one-dimensional measurements[2]. It was recognized early on that the two-dimensional nature of a real surface needed to be considered in estimating the "true" value of statistics from one-dimensional measurements, but these considerations were ignored because it was not easy to measure the 2D surface with a $1 \mathrm{D}$ profiler. The situation has now changed dramatically over the past two decades, in that 2D surface profilers have made $1 \mathrm{D}$ profilers obsolete. Now it is easy to measure $2 \mathrm{D}$ surface topography and compute $2 \mathrm{D}$ statistics in the spatial domain. But we still compute $1 \mathrm{D}$ frequency domain statistics from 2D measurements by computing the PSD of each row in the 2D array and then averaging all row PSDs into one representative PSD. This procedure still ignores the 2D nature of the surface in frequency space and leads to inconsistencies in comparing measurements between different instruments. The problem now is to reconcile statistics computed over a $2 \mathrm{D}$ spatial frequency domain between instruments, as the frequency footprint of each instrument in both the $\mathrm{x}$ - and $\mathrm{y}$-directions needs to be considered.

\section{Theoretical considerations}

The transformation from a $2 \mathrm{D}$ to a $1 \mathrm{D}$ PSD is accomplished formally by integrating the $2 \mathrm{D}$ spectrum, $\mathrm{S}_{2}\left(f_{\mathrm{x}}, f_{\mathrm{y}}\right)$ over the $f_{\mathrm{y}}$ coordinate[3]:

$$
S_{1}^{*}\left(f_{x}\right)_{\text {metsured }}=4 \int_{0}^{\infty} d f_{y} S_{2}\left(f_{x}, f_{y}\right) \cdot H\left(f_{x}, f_{y}\right)
$$

where $S_{2}$ is the two-sided PSD that is defined over positive and negative frequencies, $S_{1}{ }^{*}$ is the one-sided 1D PSD defined for positive frequencies, and $H$ is the instrument response function, which for simplicity we take to be unity here over all frequencies. Note that the value of the 1D PSD at a given frequency $f_{x}$ requires knowledge of the entire span of 2D frequencies in the y-direction at that $f_{x}$ value. In most cases, we do not know what the frequency spectrum is over this infinite range. Our knowledge is limited to a finite frequency range that depends upon the frequency range of the sampling in the spatial domain. The relationship between the sampling parameters and the frequency bandwidth is illustrated in Fig. 1. 
submitted to Optical Fabrication and Testing Topical Meeting, Optical Society of America, 14-16 June 2010, Jackson Hole, WY

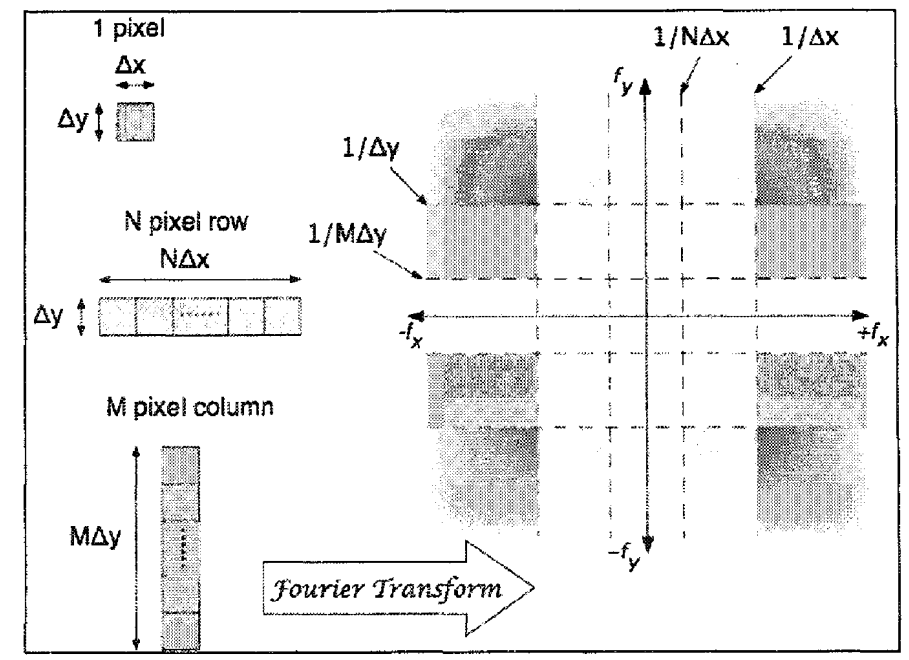

Fig. 1 - Frequency domain range for various pixel sampling conditions. A single pixel covers only the highest frequency range in the corners of frequency space, extending to infinity in both $\left|f_{x}\right|$ and $\left|f_{y}\right|$. As more pixels are added to the profile, the low frequency range approaches the origin.

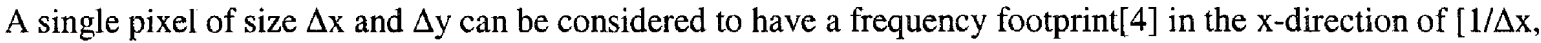
$\infty]$ and likewise in the $y$-direction of $[1 / \Delta y, \infty]$. In a profile measurement consisting of $N$ sample points in the $x-$ direction, the low frequency limit moves to $1 / \mathrm{N} \Delta \mathrm{x}$, and the frequency footprint extends to this value as shown by the yellow region in Fig. 1. Note that the y-frequency range has not changed. The same considerations show that an $\mathrm{M}$ point column profile in the $y$-direction moves the lower limit of the $y$-frequency to the lower value $1 / \mathrm{M} \Delta \mathrm{y}$.

A problem arises if we now attempt to compare the 1D roughness spectra between instruments with different spatial domain probe sizes. In order to compare 1D PSDs from both instruments, the frequency range of both instruments in the orthogonal direction must be the same. The $\mathrm{x}$-direction frequency range will, in general differ, but we assume here that there is a region of overlap between the high frequency end of the small-pixel device and the low frequency end of the large-pixel device. This is illustrated in the next section.

\section{Measurement results}

Profile measurements were made on a toroidal mirror surface with a Long Trace Profiler (LTP)[5] and a Zygo NewView 6300 Optical Profiler (NV). The LTP has a probe beam with a nominal diameter of about $1 \mathrm{~mm}$ and produces a single line profile that is about $150 \mathrm{~mm}$ in length. The $2 \mathrm{D}$ NewView measurements were made with a $640 \times 480$ pixel camera with an objective magnification of $20 \mathrm{x}$ which produce an effective pixel size of about $0.5 \mu \mathrm{m}$. The LTP is operated in a micro-sampling mode, where the step size is $0.1 \mathrm{~mm}$ per point. The surface is highly oversampled in this case and the attenuation of the high frequency signal due to averaging within the beam diameter must be considered in the analysis. This oversampling by the LTP produces a reasonable frequency overlap region between the two measurements.

The PSD curves for the two measurements are shown in Fig. 2. The uncorrected LTP curve (black) and the one row average NewView PSD (red) do not match at all in the region of overlap. This is not surprising, since the yextent of the spatial frequency ranges are so different between the machines. The effective pixel size of the LTP is about 1000 times larger than the NV, so its frequency footprint extends to much lower frequencies and the high frequencies are attenuated by averaging the height information within the beam diameter. The NV spectrum Appears to be much higher than the tail of the LTP.

Now we apply the appropriate corrections to both data sets. First, we bin the data in the NV 2D array in the vertical (column) direction to generate an effective single row profile with a frequency footprint in the y-direction that is close to that of the LTP. This averages out a significant fraction of the high frequency roughness and produces a smoother effective profile. The RMS roughness values for these two spectra are $0.57 \mathrm{~nm}$ and $0.13 \mathrm{~nm}$ integrated over the full NV bandwidth shown. When we compute the PSD from this profile, we see that the absolute magnitude of the spectrum has decreased significantly. Next, we apply a Gaussian restoration filter to the LTP spectrum with a variable sigma parameter to restore the attenuated high frequencies. Since we don't know precisely the exact LTP probe beam diameter, we use two values for the Gaussian filter, $600 \mu \mathrm{m}$ and $750 \mu \mathrm{m}$. One can see from Fig. 2 that the $600 \mu \mathrm{m}$ parameter raises the high frequency tail of the LTP so that it exactly matches the magnitude and slope of the binned NewView spectrum. 
submitted to Optical Fabrication and Testing Topical Meeting, Optical Society of America, 14-16 June 2010, Jackson Hole, WY

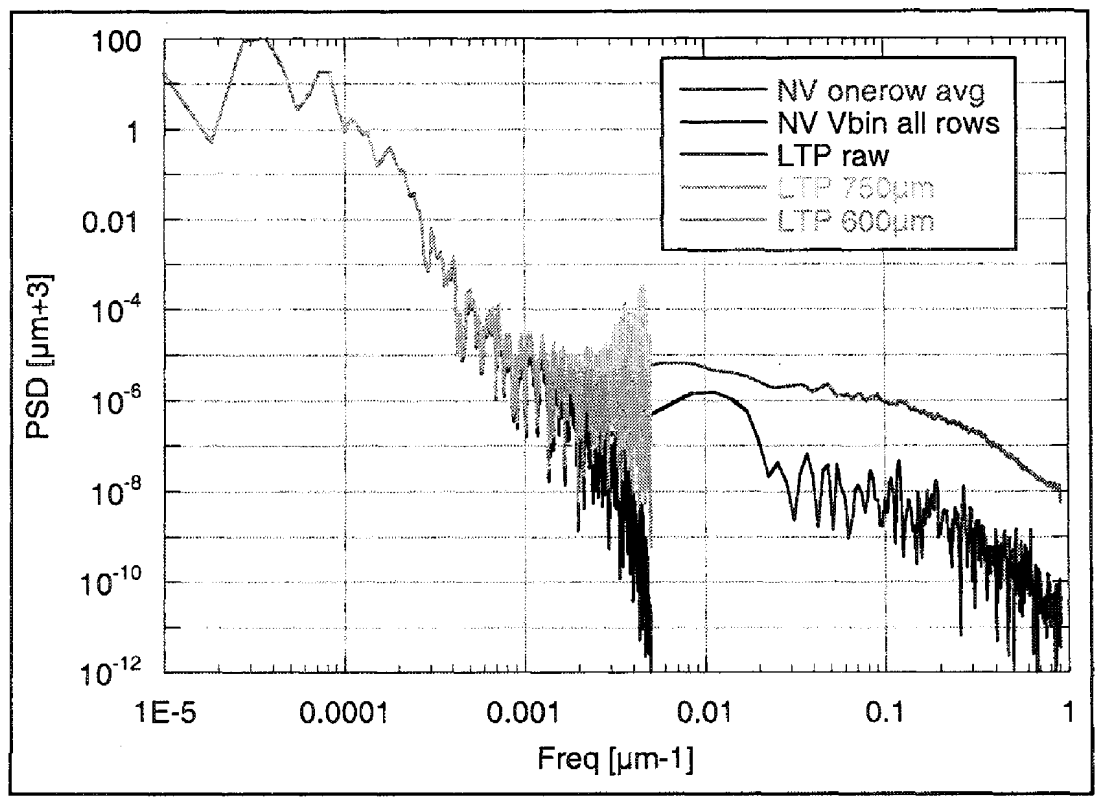

Fig. 2 -1D PSD curves for the LTP profile (left set) and the NewView data (right set.) The NewView spectra show the reduction in magnitude produced by binning the high resolution pixels in the vertical direction to match the low frequency footprint of the LTP in the y-direction. A Gaussian restoration filter applied to the LTP spectrum boosts up the tail of the curve to match the magnitude and slope of the New View spectrum

\section{Conclusions}

This crude analysis indicates that it is necessary to account for the precise spatial frequency footprint of each instrument in the orthogonal direction when comparing 1D spectra from different instruments. This caveat applies also to measurements made by the same instrument with different magnification objectives. That the RMS roughness statistics can differ by such a large factor, $0.57 \mathrm{~nm}$ vs. $0.13 \mathrm{~nm}$, indicates that it may not be sufficient to specify just the $x$-direction spatial frequency range in the statistical quantities derived from 1D PSD calculations. An effort is underway to provide a more rigorous analytic description of this problem.

\section{Acknowledgements}

This manuscript has been authored by Brookhaven Science Associates, LLC under Contract No. DE-AC0298CH10886 with the U.S. Department of Energy. The United States Government retains, and the publisher, by accepting the article for publication, acknowledges, a world-wide license to publish or reproduce the published form of this manuscript, or allow others to do so, for the United States Government purposes. The Advanced Light Source is supported by the Director, Office of Science, Office of Basic Energy Sciences, Material Science Division, of the U.S. Department of Energy under Contract No. DE-AC02-05CH11231 at Lawrence Berkeley National Laboratory.

\section{Disclaimer}

Certain commercial equipment, instruments, or materials are identified in this document. Such identification does not imply recommendation or endorsement by the US Department of Energy, BNL, LBNL, or ALS, nor does it imply that the products identified are necessarily the best available for the purpose.

\section{References}

[1] Church, E.L. and Berry, H.C., Spectral analysis of the finish of polished optical surfaces, Wear 83, p. 189-201 (1982).

[2] Bhushan, B., Wyant, J.C., and Koliopoulos, C.L., Measurement of surface topography of magnetic tapes by Mirau interferometry, Applied Optics 24, p. 1489-1497 (1985).

[3] Church, E.L. and Takacs, P.Z., Chapter 8 - Surface Scattering, in Handbook of Optics - 3rd edition, M. Bass, Editor. McGraw-Hill. (2010) [4] Church, E.L., The precision measurement and characterization of surface finish in "Precision Surface Metrology", Proc. SPIE 429, pp. 86-95, (1983)

[5] Takacs, P.Z., Furenlid, K., DeBiasse, R., and Church, E.L., "Surface topography measurements over the 1 meter to 10 micrometer spatial period bandwidth", in Surface Characterization and Testing II, Proc. SPIE 1164, eds. J.E. Grievenkamp and M. Young, pp. 203-211, (1989). 